\title{
A FEW EXAMPLES AND COUNTEREXAMPLES IN SPECTRAL GRAPH THEORY
}

\author{
Dragan Stevanović \\ Mathematical Institute \\ Serbian Academy of Sciences and Arts, Belgrade, Serbia \\ e-mail: dragance106@yahoo.com \\ Nikola Milosavluević \\ University of Niš \\ Faculty of Sciences and Mathematics, Niš, Serbia \\ e-mail: nikola5000@gmail.com \\ AND \\ DAMIR VUKIČEVIĆ \\ University of Split \\ Faculty of Sciences and Mathematics, Split, Croatia \\ e-mail: vukicevi@pmfst.hr
}

Dedicated to the memory of Professor Slobodan Simić.

\begin{abstract}
We present a small collection of examples and counterexamples for selected problems, mostly in spectral graph theory, that have occupied our minds over a number of years without being completely resolved.

Keywords: communicability distance, spectral radius, integral graph, second Zagreb index, Wiener index, estrada index, almost cospectral graphs, NEPS of graphs.
\end{abstract}

2010 Mathematics Subject Classification: 05C50. 
[1] R. Askey, Evaluation of Sylvester type determinants using orthogonal polynomials, in: Proceedings of the 4th International ISAAC Congress, Toronto, Canada, August 11-16, 2003, Advances in Analysis, H.G.W. Begehr et al. (Ed(s)), (World Scientific, 2005) $1-16$.

doi:10.1142/9789812701732_0001

[2] V. Brankov, P. Hansen and D. Stevanović, Automated conjectures on upper bounds for the largest Laplacian eigenvalue of graphs, Linear Algebra Appl. 414 (2006) 407-424. doi:10.1016/j.laa.2005.10.017

[3] L. Chen and Q. Huang, The existence of the graphs that have exactly two main eigenvalues (2016). arXiv:1609.05347

[4] S.M. Cioaba and D.A. Gregory, Principal eigenvectors of irregular graphs, Electron. J. Linear Algebra 16 (2007) 366-379. doi:10.13001/1081-3810.1208

[5] D.M. Cvetković, The main part of the spectrum, divisors and switching of graphs, Publ. Inst. Math. (Beograd) (N.S.) 23(37) (1978) 31-38.

[6] D. Cvetković, Discussing graph theory with a computer II. Theorems suggested by the computer, Publ. Inst. Math. (Beograd) (N.S.) 33(47) (1983) 29-33.

[7] D. Cvetković, On the 2-sum of three graphs. Variations on the graph product disconnectedness theme, Bull. Cl. Sci. Math. Nat. Sci. Math. 24 (1999) 107-117.

[8] D. Cvetković, M. Doob and H. Sachs, Spectra of Graphs-Theory and Application (Academic Press, New York, 1980).

[9] D. Cvetković and R. Lučić, A new generalization of the concept of the p-sum of graphs, Univ. Beograd. Publ. Elektrotehn. Fak. Ser. Mat. Fiz. 302-319 (1970) $67-71$.

[10] D. Cvetković, P. Rowlinson and S. Simić, An Introduction to the Theory of Graph Spectra (Cambridge University Press, Cambridge, 2009). doi:10.1017/CBO9780511801518

[11] K.Ch. Das and M.J. Nadjafi-Arani, On maximum Wiener index of trees and graphs with given radius, J. Comb. Optim. 34 (2017) 574-587. doi:10.1007/s10878-016-0092-y

[12] C. Elphick and T. Réti, On the relations between the Zagreb indices, clique numbers and walks in graphs, MATCH Commun. Math. Comput. Chem. 74 (2015) 19-34.

[13] E. Estrada, The communicability distance in graphs, Linear Algebra Appl. 436 (2012) 4317-4328. doi:10.1016/j.laa.2012.01.017

[14] X. Fan, Y. Luo and X. Gao, Tricyclic graphs with exactly two main eigenvalues, Open Math. 11 (2013) 1800-1816.

doi:10.2478/s11533-013-0283-z 
[15] M. Ghebleh, A. Kanso and D. Stevanović, Graph6 Java: a researcher-friendly Java framework for testing conjectures in chemical graph theory, MATCH Commun. Math. Comput. Chem. 81 (2019) 737-770.

[16] E.M. Hagos, Some results on graph spectra, Linear Algebra Appl. 356 (2002) 103-111. doi:10.1016/S0024-3795(02)00324-5

[17] S. Hayat, J.H. Koolen, F. Liu and Z. Qiao, A note on graphs with exactly two main eigenvalues, Linear Algebra Appl. 511 (2016) 318-327.

doi:10.1016/j.laa.2016.09.019

[18] O. Holtz, Evaluation of Sylvester type determinants using block-triangularization, in: Proceedings of the 4th international ISAAC congress, Toronto, Canada, August 11-16, 2003, Advances in Analysis, H.G.W. Begehr et al. (Ed(s)), (World Scientific, 2005) 395-405. doi:10.1142/9789812701732_0036

[19] Y. Hou, Z. Tang and W.C. Shiu, Some results on graphs with exactly two main eigenvalues, Appl. Math. Lett. 25 (2012) 1274-1278. doi:10.1016/j.aml.2011.11.025

[20] Y. Hou and F. Tian, Unicyclic graphs with exactly two main eigenvalues, Appl. Math. Lett. 19 (2006) 1143-1147. doi:10.1016/j.aml.2005.11.025

[21] Z. Hu, S. Li and C. Zhu, Bicyclic graphs with exactly two main eigenvalues, Linear Algebra Appl. 431 (2009) 1848-1857. doi:10.1016/j.laa.2009.06.022

[22] X. Huang, Q. Huang and L. Lu, Construction of graphs with exactly $k$ main eigenvalues, Linear Algebra Appl. 486 (2015) 204-218. doi:10.1016/j.laa.2015.08.013

[23] T. Muir, The Theory of Determinants in the Historical Order of Development, 2nd Edition (Macmillan and Co., London, 1906).

[24] S. Mukwembi and T. Vetrík, Wiener index of trees of given order and diameter at most 6, Bull. Austral. Math. Soc. 89 (2014) 379-396. doi: $10.1017 /$ S0004972713000816

[25] V. Nikiforov, Walks and the spectral radius of graphs, Linear Algebra Appl. 418 (2006) 257-268. doi:10.1016/j.laa.2006.02.003

[26] J. Plesnik, On the sum of all distances in graph or diagraph, J. Graph Theory 8 (1984) $1-21$. doi:10.1002/jgt.3190080102

[27] P. Rowlinson, The main eigenvalues of a graph: A survey, Appl. Anal. Discrete Math. 1 (2007) 445-471. doi:10.2298/AADM0702445R 
[28] S. Simić, I. Gutman and V. Baltić, Some graphs with extremal Szeged index, Math. Slovaca 50 (2000) 1-15.

[29] L. Shi, On graphs with given main eigenvalues, Appl. Math. Lett. 22 (2009) 18701874 .

doi:10.1016/j.aml.2009.06.027

[30] W. So, Integral circulant graphs, Discrete Math. 306 (2006) 153-158.

doi:10.1016/j.disc.2005.11.006

[31] D. Stevanović, When can the components of NEPS of connected bipartite graphs be almost cospectral?, Linear Algebra Appl. 311 (2000) 35-44.

doi:10.1016/S0024-3795(00)00061-6

[32] D. Stevanović, On the components of NEPS of connected bipartite graphs, Linear Algebra Appl. 356 (2002) 67-78. doi:10.1016/S0024-3795(02)00322-1

[33] Z. Tang and Y. Hou, The integral graphs with index 3 and exactly two main eigenvalues, Linear Algebra Appl. 433 (2010) 984-993.

doi:10.1016/j.laa.2010.04.025

[34] H. Täubig, Matrix Inequalities for Iterative Systems (CRC Press, Boca Raton, 2017). doi:10.1201/9781315166131

Received 26 April 2019

Revised 26 August 2019

Accepted 28 August 2019 